\title{
LA IMPORTANCIA DE LOS EXPERIMENTOS MENTALES EN LA ENSEÑANZA DE LA FÍSICA
}

\author{
The importance of thought experiments in the teaching of \\ Physics
}

\author{
Vicente Menéndez \\ ORCID http://orcid.org/0000-0003-0199-1291 \\ Instituto Superior de Formación Docente $N^{\circ} 117$, San Fernando, Provincia de Buenos Aires, \\ Argentina.
}

Recibido: 31 de agosto de 2020 Aceptado: 22 de diciembre de 2020

\section{Resumen}

Ernest Mach sugirió en el siglo XIX introducir en el aula los experimentos mentales ${ }^{1}$ y fue Einstein quien situaría los experimentos mentales, en el escenario principal de la física moderna ${ }^{2}$. Desarrollaremos en el presente trabajo, la descripción de algunas experiencias mentales (en adelante EM) para trabajar con los alumnos, porque pensamos que no solo serán una ayuda didáctica importante, sino que los casos que trataremos, son de importancia conceptual.

Palabras clave: Enseñanza, Física, Métodos

\begin{abstract}
Ernest Mach suggested introducing thought experiments into the classroom in the nineteenth century and it was Einstein who would place thought experiments on the main stage of modern physics. In this work we will develop the description of some mental experiences (here in after EM) to work with the students, because we think that they will not only be an important didactic aid, but that the cases that we will deal with are of conceptual importance.
\end{abstract}

Keywords: Teaching, Physics, Methods

\section{Introducción}

Quizá Aristóteles haya sido el iniciador de los llamados “experimentos mentales”, en la física, al decir que dos cuerpos de distinto peso, caen con velocidades proporcionales a los mismos ${ }^{3}$ Y este es quizás (resultado de la intuición) el experimento mental más fatídico de la historia de la Física, que hizo decir a Bertrand Rusell, con una concepción anacrónica de la historia de la ciencia, que Aristóteles fuese uno de los grandes infortunios de la raza humana ${ }^{4}$. Por la contraria, y con una lógica apartada de la intuición, y del "sentido común", fue Galileo quien realizó el primero de los experimentos mentales más fructíferos, lo llamaremos Experiencia Mental 1.

\footnotetext{
1 Matthews, pág. 228

2 Ibíd. pág. 228

3 Boido, G. Págs 36 y 37

$4 \quad$ Bertrand Rusell, pág. 36
} 


\section{EM 1: Principio de Inercia}

Dice el historiador de la ciencia A. Koyrés:

"El principio de inercia no salió ya elaborado del pensamiento de Descartes o de Galileo como Atenea de la cabeza de Zeus. La formación del nuevo concepto de movimiento- que implica un nuevo concepto de la realidad física- del que el principio de inercia es, a la vez expresión y sostén, fue precisada por un largo y penoso trabajo mental"

Fue Newton en sus Principios matemáticos de la filosofía natural, quien enunció el principio de Inercia tal como hoy lo conocemos. Un principio imposible de comprobar experimentalmente: cómo podemos verificar el movimiento rectilíneo y uniforme hasta el infinito? Podemos decir entonces, que la nueva física surgida con Galileo, nace de un experimento mental.

\section{EM 2. Caída de los cuerpos}

La caída de los cuerpos es un buen ejemplo de EM para trabajar con los alumnos, dado que en general, y probablemente como producto de la intuición, se piensa que el objeto más pesado, cae antes que uno liviano, si son arrojados al mismo tiempo.

En su ya célebre trabajo La lógica de la investigación científica, Karl Popper, dice ${ }^{6}$

Uno de los experimentos imaginarios más importantes de la historia de la filosofía natural, y uno de los argumentos más sencillos y más ingeniosos de la historia del pensamiento racional acerca de nuestro universo, están contenidos en la crítica de Galileo de la teoría del movimiento aristotélico.

Lo que dice Popper puede leerse en la Jornada primera de las "Consideraciones y demostraciones matemáticas sobre dos nuevas ciencias”, publicado en 1638, (o sea después del Juicio y con Galileo en prisión). Allí transcurre un dialogo entre dos personajes, Salviati, que representa el pensamiento de Galileo, y el aristotélico Simplicio7, respecto de la caída libre de los cuerpos:

Salviati. Entonces, si nosotros tuviéramos dos móviles, cuyas velocidades naturales fuesen distintas, es evidente que si uniésemos ambos, el más rápido perdería velocidad por obra del más lento, mientras que este aceleraría debido al más rápido. Estáis de acuerdo con lo que acabo de decir?

Simplicio. Me parece que las cosas deben, ciertamente, suceder así. 


\begin{abstract}
Salviati. Pero si esto es así, y si es verdad, por otro lado, que una piedra grande se mueve, por ejemplo, con una velocidad de 8 grados y una piedra pequeña, con una velocidad de 4, si las uniésemos, el resultado de ambas, según lo dicho, será inferior a 8 grados de velocidad. Ahora bien, las dos piedras juntas dan por resultado una más grande que la primera que se movía con 8 grados de velocidad; de lo que se sigue que tal compuesto se moverá a más velocidad que la primera de las piedras sola, lo cual contradice vuestra hipótesis. Veis, pues, como suponiendo que el móvil más pesado se mueve a más velocidad que el que pesa menos. Concluyo que el más pesado se mueve a menos velocidad.
\end{abstract}

Simplicio Yo me encuentro completamente ofuscado...

Finalmente diremos, que la muy popular imagen de Galileo arrojando los cuerpos desde lo alto de la torre de Pisa, para demostrar experimentalmente la ley de caída de los cuerpos, ha sido cuestionada en su veracidad por algunos historiadores de la ciencia, en particular por Alexander Koyre. En su trabajo Estudios de historia del pensamiento científico, en el capítulo: Galileo y el experimento de Pisa: a propósito de una leyenda8.

Por otro lado expresa Koyre, que por suerte no lo realizó, pues de hacer la experiencia (por efecto del empuje en el aire y la fuerza de rozamiento), se vería tocar primero el suelo, al más pesado.

El ejemplo citado, permite además introducir la cuestión histórica, que suelen ser un aporte cultural interesante.

\title{
EM 3. Principio de Arquímedes
}

Suele realizarse la demostración del principio de Arquímedes, sumergiendo un objeto en un recipiente lleno de líquido; pesando el líquido derramado, se obtiene, que el valor del peso del cuerpo sumergido, es igual al peso del cuerpo en el aire menos el peso del líquido derramado.

Pero se puede hacer el EM de una forma muy simple: supongamos un recipiente con un líquido en reposo y aislemos mentalmente una esfera dentro de ese líquido. Dicha esfera está por supuesto en equilibrio, o sea que sobre esa esfera imaginaria, que tiene un determinado peso, debe actuar sobre ella una fuerza contraria al peso, que llamaremos Empuje, y que debe tener el mismo valor que el peso de dicha esfera, para estar, como evidentemente lo está, en reposo. O sea que esa fuerza Empuje es igual al peso del líquido desalojado por dicha esfera imaginaria. Y esto no es otra cosa que el enunciado del famoso principio. Digamos entonces que para llegar a él, no hace falta ninguna experiencia, la cual en todo caso, serviría para corroborar el Principio, que generalmente se enseña simplemente definiéndolo, o a través de la experiencia. Pero con esta EM se comprende el verdadero fundamento físico del mismo, cosa que no podía saber Arquímedes pues no disponía de las leyes de la dinámica (la sumatoria de las fuerzas debe ser nula para que un cuerpo se halle en equilibrio). 


\section{EM 4. Ley de la refracción}

La conocida Ley de Snell o de la refracción de la luz, que establece, que la relación entre el seno del ángulo de incidencia y el seno del ángulo de refracción es una constante, denominada índice de refracción, fue hallada experimentalmente por varios filósofos naturales: el francés Descartes, el holandés Snell, y el inglés Harriot. Incluso Ptolomeo en el siglo II d.C realizó la experiencia, aunque sin éxito, para hallar dicha ley ${ }^{9}$. Investigaciones recientes hallaron que los árabes en el siglo X llegaron a establecer la ley tal como hoy la conocemos ${ }^{10}$.

Pero hubo un matemático francés, Pierre de Fermat (1601-1665) célebre por su famoso último teorema, que pudo ser resuelto recién en 1993, quien realizó una de las EM más interesantes en la historia de la física ${ }^{11}$ : imaginemos una playa y un guardavida ubicado en A y un bañista en B (ver Fig.1)

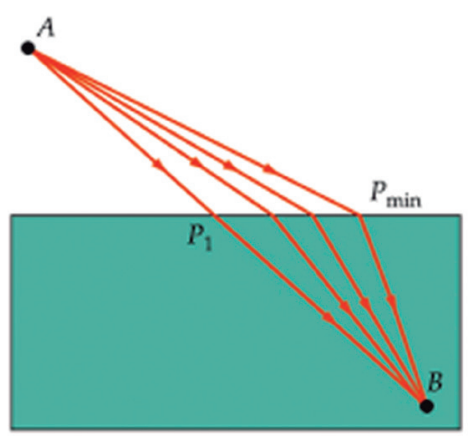

Figura 1

En estas circunstancias, se pregunta Fermat, que camino hará el guardavida para rescatar a B: siguiendo la trayectoria más corta entre A y B, o sea correr hasta P1 y luego nadar de P1 a B, o correr hasta Pmin y luego nadar hasta B? Elige esta última trayectoria, que si bien no es la más corta, es la que Fermat supone requiere menor tiempo. Y esto es lo que precisamente hace la luz, va por el camino de tiempo mínimo, pues la velocidad de la luz en el aire es mayor que en el agua (fue recién en 1849 cuando H. Fizeau realizó el experimento para medir la velocidad de la luz en el agua). A esto se lo conoce como Principio de Fermat. Se puede demostrar fácilmente (considerando el frente de ondas propuesto por Huygens en su teoría ondulatoria de la luz) que la relación entre los senos de los ángulos de incidencia y de refracción, son iguales al cociente entre las velocidades de la luz en el aire y en el otro medio, por ejemplo el agua, siendo este cociente entre dichas velocidades, igual en valor al índice de refracción hallado experimentalmente. Ello da, además como consecuencia, que la velocidad de la luz en el aire es mayor que en el agua, inversamente a lo postulado por Newton en su teoría corpuscular de la luz, (al considerar a la luz compuesta por corpúsculos, considera que los mismos son atraídos por las partículas del agua y por lo tanto incrementarán su velocidad). Conclusión: sin realizar ninguna experiencia, Fermat encuentra a través de una EM un principio fundamental, que también podemos sintetizar así: la naturaleza es económica en su actuar. 


\section{EM 5 Las experiencias mentales de Newton}

Como dice Matthews, "Además de su trabajo experimental con péndulos y prismas, la ciencia de Newton, dependían en gran medida de experimentos mentales, algo que reconoció Ernst Mach, pero que los maestros de ciencia modernos no reconocen con tanta frecuencia ${ }^{12}$.

Uno de los EM mas famosos de Newton, es aquel en que supone una piedra, que arrojada cada vez con mas velocidad, caerá mas lejanamente, hasta llegar a una velocidad tal (que hoy llamamos velocidad de escape) que su caída sea la misma que la curvatura terrestre, en cuyo caso la piedra se convertiría en un satélite artificial de la Tierra. Esto está expuesto en El sistema del mundo ${ }^{13}$. La importancia de tal EM radica en la unificación de la física terrestre con la celeste, lo cual es necesario para entender la atracción gravitatoria universal.

Por supuesto que las abstracciones geniales de Newton son varias, entre ellas considerar el principio de acción y reacción no solo para las acciones de contacto, sino también para las fuerzas a distancia. En el caso de este principio hay una EM interesante para trabajar con los alumnos: que pasa cuando una botella tapada con una mosca en la base se encuentra en equilibrio sobre una balanza, y la mosca sale volando. ¿Pesará menos cuando la mosca está volando?

\section{EM 6 Las experiencias mentales de Einstein}

Si hay un científico que recurrió innumerables veces a las EM, ese científico fue Albert Einstein. Demostrando una vez más la importancia de los mismos, y también, en cuanto a la cuestión epistemológica, en como la ciencia se ha construido en gran medida a través del método hipotético -deductivo, quizás en mayor medida que por el método experimental inductivo. En varias oportunidades, Einstein comentó, que el día más feliz de su vida fue cuando se imaginó cayendo en el vacío, y sin tener ninguna referencia cercana: en ese caso no sentiría el efecto de la gravedad, la cual fue una de las ideas que lo llevó a desarrollar la teoría general de la relatividad. En otra ocasión, dijo que lo inquietaba la interpretación física de las ecuaciones de Maxwell, y que "esta sensación desapareció gracias a un experimento mental: se imaginó corriendo junto a un rayo de luz y volteando a mirarlo, lo vio como un campo electromagnético oscilatorio en reposo espacialmente. Pero al parecer no existe tal cosa ya sea según la experiencia o de acuerdo con las ecuaciones de Maxwell. Se afirma que esta paradoja contenía el germen de la teoría especial de la relatividad ${ }^{14}$.

Un maravilloso ejemplo de cómo, tan solo con la mente, se puede descubrir secretos de la naturaleza, son las famosas aporías de Zenón de Elea, hechas cinco siglos antes de Cristo. Una de ellas es la demostración de que el espacio absoluto no existe. Dice Zenón: si todo lo que existe está en el espacio, entonces éste para existir, debe estar contenido en otro espacio y así sucesivamente, deberían existir infinitos espacios, lo cual es un absurdo ${ }^{15}$. Conclusión, el espacio absoluto no existe. Tal como afirma la Teoría General de la Relatividad, postulada 2400 años después.

\footnotetext{
12 Matthews, pág. 222

13 Newton, pág. 49

14 Matthews, Pág. 226

15 AranaJ. Pág. 63
} 
Durante el período crítico de discusiones, respecto a la interpretación física de los nuevos desarrollos de la teoría cuántica, Einstein volvió a plantear experiencias imaginarias, tratando de refutar las consecuencias del Principio de incertidumbre, pero en este caso con ejemplos que fueron refutados en su momento por Niels Bohr.

\section{Conclusiones}

Con estos pocos ejemplos históricos, hemos tratado de demostrar lo importante de introducir los experimentos mentales en la enseñanza de la ciencia, siendo esto una ayuda didáctica que estimula la imaginación en los alumnos. Y demuestran también, que no solo en el laboratorio se construye la ciencia.

\section{Bibliografía}

Arana, J (2001). Materia, Universo, Vida. Ed. Tecnos, Madrid

Boido, G (1998). Noticias del planeta Tierra. Galileo Galilei y la revolución científica. AZ Editora, Buenos Aires

Chalmers, A (1992). La ciencia y como se elabora. Siglo XXI, Madrid

Galileo, G (1996). Consideraciones y demostraciones matemáticas sobre dos nuevas ciencias. Ed. Planeta De Agostini S.A. Barcelona

Guizal, B. y Dudley, J. (2003). Ibn Sahl, descubridor de la ley de la refracción. Revista Investigación y Ciencia, $\mathrm{N}^{\circ} 317$

Koyre, A (1990). Estudios de historia del pensamiento científico Siglo XXI, Madrid Koyré, A (1998). Estudios Galileanos, Siglo XXI, Madrid

Loe del Palumbo, E (1957). Física elemental. Editorial Estrada, Buenos Aires Matthews, M (2017). La enseñanza de la ciencia. F.C. E. México

Newton, I. (1992). El sistema del mundo Alianza Editorial, Madrid

Popper, K (1977). La lógica de la investigación científica Ed. Tecnos, Madrid

Russell, B (1982). La perspectiva científica. Ed. Ariel, Barcelona 